EPJ Web of Conferences 19, 05011 (2012)

DOI: $10.1051 /$ epjconf/20121905011

(C) Owned by the authors, published by EDP Sciences, 2012

\title{
Large scale characterization of the stellar velocity distribution in the galactic disk
}

\author{
S. Roca-Fàbrega ${ }^{1, a}$, O. Valenzuela ${ }^{2}$, F. Figueras ${ }^{1}$, M. Romero-Gómez ${ }^{1}$ \\ and T. Antoja ${ }^{3}$
}

1 ICCUB-IEEC, Universitat de Barcelona

2 Instituto de Astronomía, UNAM

${ }^{3}$ Kapteyn Astronomical Institute, Groningen

\begin{abstract}
Test particle simulations of Milky Way type galactic disks are being conducted to map the evolution of the stellar large scale kinematic response to the bar and spiral structure. Second and third order moments of the velocity distribution function prove to be good indicators of both, the velocity ellipsoid misalignment near the arms and the bar, and the degree of kinematic substructure in the UV plane, that is, the presence of moving groups. A large scale analysis all through the galactic disk allows to establish the kinematic behavior near resonances and the correlation between the kinematic parameters and properties of the non-axisymmetric components, such as its transient nature or its mass overdensity. N-body simulaions are being run in order to test these results in self-consistent models.
\end{abstract}

The study of the origin and evolution of moving groups in the Milky Way (MW) disk has been up to now mostly restricted to the solar radius [1]. Work is being conducted to characterize the kinematics all throughout the disk by second and third order moments of the velocity distribution. For that we use both N-body and test particle simulations.

Test particle simulations consider an Allen \& Santillán [2] axisimetric potential that consist of a bulge, disk and halo whose rotation curve is similar to the MW's, and a prolate bar [3]. We integrate the particles during $400 \mathrm{Myr}$, and we analyse the kinematics of the particles at this final snapshot. In particular, we compute the second and the third ordermoments of the velocity distribution function, and with the first ones, the vertex deviation.

Our simulations confirm that the vertex deviation is a good tracer of the spiral perturbation [4], showing a clear variation near spiral arm's overdensity (see figures 1 and 2). This is consistent with the results of [5], although in our case the spiral structure has not been imposed, that is, the spiral arms are the response to the bar potential. Work is in progress to evaluate the capabilities of the vertex deviation paremeter to trace the regions of the Galactic disc where particles present a kinematic substructure similar to the one observed in the Solar neighbourhood [6].

Radial and azimuthal third order moments of the velocity distribution function are potentially good candidates to trace the degree of substructre, too. As espected, they additionally trace the main properties of the galactic potential.

Currently, we are running a set of n-body simulations to test these results in a self-consistent models. In particular we observe that the radial third order moments follow the same pattern in both, test-particle and N-body simulations. On the other hand, the distribution of azimutal third order moments through the plane differ considerably from n-body to test-particle simulations.

\footnotetext{
a e-mail: sroca@am.ub.es
}

This is an Open Access article distributed under the terms of the Creative Commons Attribution-Noncommercial License 3.0, which permits unrestricted use, distribution, and reproduction in any noncommercial medium, provided the original work is properly cited. 

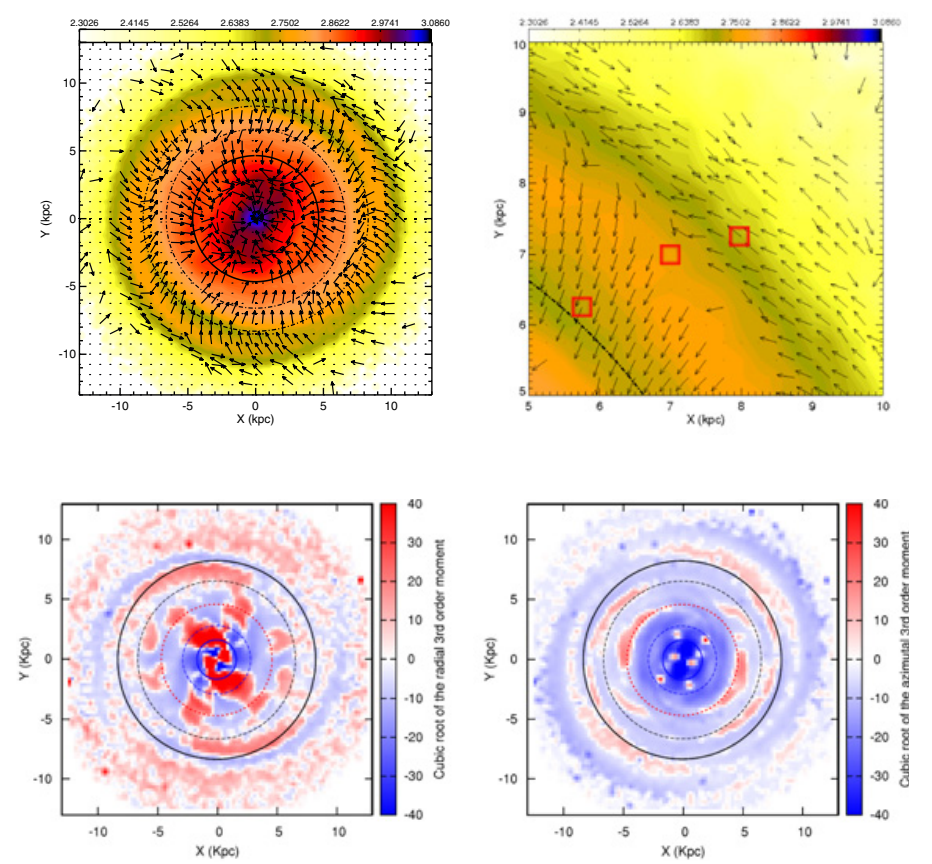

Figure 1. Up panels: Semi-major axes of velocity ellipsoid (arrows) and density (background colours), calculated for a circular regions of $500 \mathrm{pc}$ radius, of the test-particle simulation. Left panel show the analysis for all disk. Right panel shows the analysis for an arm-interarm region. X, Y axes are distance in Kpc, and colour bar the logarithm of number of particles in each region. Red boxes indicate the location of U-V plane regions. Bottom panels: Radial (left) and azimuthal (right) third order moments of the velocity DF, the colour scale shows the value of this variables. The circles indicate the bar's ILR (4:1 and 2:1) in blue, OLR (4:1 and 2:1) in black and corrotation ressonances in red.
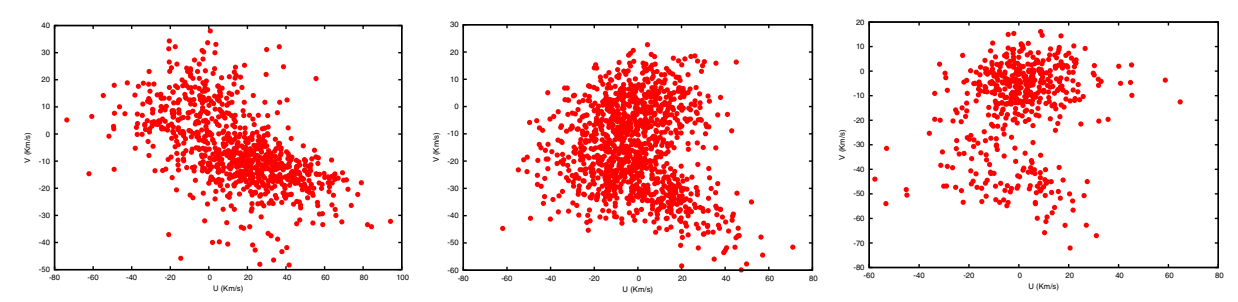

Figure 2. U-V galactocentric velocity planes of three disk regions (red boxes in top right panel of figure 1). Left panel corresponds to an inter-arm region near OLR 4:1, middle panel corresponds to an arm region and right panel to a region at the end of the arm's overdensity.

For n-body simulations we obtained an initial conditions using Hernquist method, like in [7], with one million particles in the disk, where each one have a mass of $5 \cdot 10^{4} M_{\odot}$, a $\mathrm{Q}$ parameter initially constant all above the disk $(\mathrm{Q}=1.2)$, an dark matter halo of $1.38 \cdot 10^{12} M_{\odot}$, and a $11 \mathrm{Kpc}$ spatial resolution.

\section{References}

[1] Antoja, T., et al., ApJ 700 (2009) 78

[2] Allen, C., \& Santillan, A., RMxAA 22 (1991) 255A 
Assembling the Puzzle of the Milky Way

[3] Pichardo, B., et al., ApJ 609 (1972) 144

[4] Mayor, M., A\&A 18 (1972) 97

[5] Theis, C., Vorobyov, E., chas.book 105 (2009)

[6] Antoja, T., Figueras, F., Fernández, D., Torra, J., A\&A 490 (2008) 135

[7] Klypin, A., Valenzuela, O., Colin, P., Quinn, T., MNRAS 398 (2009) 1027 